\title{
EQUILIBRIUM OF MULTIVALUED PERMANENT SYSTEMS WITH NONCONVEX RIGHT-HAND SIDES
}

\author{
BY \\ LI YONG, WANG HUAIZHONG, AND LU XIANRUI \\ Department of Mathematics, Jilin University, P. R. China
}

\begin{abstract}
The existence of periodic solutions for differential inclusions with nonconvex right-hand sides is proved. As an application, an affirmative answer to the existence of an equilibrium for permanent multivalued systems with nonconvex righthand sides is given.
\end{abstract}

1. Introduction. Deterministic modelling in the biological sciences often leads to ordinary differential equations defined on a certain state space,

$$
x^{\prime}=f(x),
$$

for example ecological differential equations with the state space $\mathbf{R}_{+}^{n}$ (the positive cone):

$$
x_{i}^{\prime}=x_{i} f_{i}(x), \quad i=1, \ldots, n, x=\left(x_{1}, \ldots, x_{n}\right) .
$$

For such equations, an important and interesting problem is whether permanence implies the existence of an equilibrium state or not.

Here (1) is said to be permanent if there exist $m, M \in(0, \infty)$ such that, given any $x \in$ int $\mathbf{R}_{+}^{n}$ (the interior of $\mathbf{R}_{+}^{n}$ ), there is a $T_{x}$ such that every solution $x(t)$ starting at $x$ satisfies

$$
m \leq x_{i}(t) \leq M, \quad i=1, \ldots, n, t \geq T_{x} .
$$

Biologically, permanence ensures the long-term survival of all species. There has been a large amount of work devoted to this topic (see, for example, $[3,5,7,12-$ 14, 16-19]). As argued in [17], it is reasonable to expect a positive answer to the existence question above. This has indeed been proved by Hofbauer and Sigmund [14], Hutson and Moran [18], and Hutson [17]. In particular, in [17] Hutson gives a simple and efficient discussion of the problem by using the asymptotic Schauder fixed point theorem. There he also proposed the following open problem:

For the multivalued system

$$
x^{\prime} \in F(x),
$$

does permanence imply existence of an equilibrium state?

Received March 1, 1993.

1991 Mathematics Subject Classification. Primary 34A60, 34C25. 
When $F(x)$ is a compact convex-valued upper semicontinuous map, this problem has been positively solved in our paper [21], recently, by utilizing the asymptotic Schauder fixed point theorem and the approximation theorem of multivalued maps due to Zaremba (see [1] and [28]). However, for the case in which $F(x)$ is not necessarily convex valued, the problem remains open, and we would like to discuss it in the present paper.

As a general observation, we consider the existence problem of periodic solutions for differential inclusions of the form

$$
x^{\prime} \in f(t, x)
$$

where $f$ is a multivalued map from $\mathbf{R} \times X$ into $X$ with a real separable Banach space. The problem has been investigated by many authors; see, for example, [8-10, $20,23,25]$.

Here we are concerned with the following problem:

If solutions of (4) are uniformly bounded and uniformly-ultimately bounded, does (4) have periodic solutions?

For ordinary or functional differential equations satisfying the uniqueness condition with respect to initial values, such results have been established by Yoshizawa [27], Hale and Lopes [11], Burton and Zhang [4]. However, solutions of (4) with respect to initial values are not necessarily unique, or convex valued. Hence, direct applications of asymptotic fixed point theorems, due to Browder [2], Nussbaum [24], and Horn [15], to the Poincare map of (4) seem to be impossible. To overcome these difficulties, we exploit a continuous selection theorem due to Colombo, Fryszkowski, Rzežuchowski, and Staicu [6]. Of course, in our discussion, some additional conditions on $F(x)$ and $f(t, x)$ are required.

The plan of the paper is as follows. In Sec. 2, using the continuous selection theorem and Horn's asymptotic fixed point theorem (see [15]), we prove that under certain conditions, the uniform boundedness and uniform-ultimate boundedness of solutions for (4) imply the existence of periodic solutions; then applying this result, we give an affirmative answer to the equilibrium problem of (3) in Sec. 3.

2. Periodic solutions. The aim of this section is to present a general existence theorem on periodic solutions of (4).

First, let us recall some basic concepts, definitions and facts (see [26]).

Let $X$ be a real separable space with norm $|\cdot|$, and let $\operatorname{Comp}(X)$ denote the metric space of nonempty compact subsets of $X$ with the Hausdorff metric $h$ defined by

$$
h(A, B)=\max \left\{\sup _{x \in B} \operatorname{dist}(x, A), \sup _{x \in A} \operatorname{dist}(x, B)\right\} .
$$

Let $\omega>0$ and denote by $\mathscr{L}$ the $\sigma$-algebra of all Lebesgue measurable subsets of $[0, \omega]$ and by $\mathscr{B}(X)$ the family of all Borel subsets. By $C([0, \omega], X)$ (resp. $\left.L^{1}([0, \omega], X)\right)$ we mean the Banach space of all continuous (resp. Bochner integrable) maps $x:[0, \omega] \rightarrow X$ endowed with norm $\|x\|=\sup \{|x(t)|: t \in[0, \omega]\}$ (resp. $\left.\|x\|_{1}=\int_{0}^{\omega}|x(t)| d t\right)$. $\mathrm{AC}([0, \omega], X)$ stands for the Banach space of all absolutely continuous maps $x:[0, \omega] \rightarrow X$ with norm $\|x\|_{\mathrm{AC}}=|x(0)|+\left\|x^{\prime}\right\|_{1}$. 
Let $S$ be a separable metric space and let $\mathscr{A}$ be a $\sigma$-algebra of subsets of $S$. A map $G: S \rightarrow \operatorname{Comp}(X)$ is said to be $\mathscr{A}$-measurable if for each closed subset $C$ of $X$, the set $\{s \in S: G(s) \cap C \neq \varnothing\} \in \mathscr{A}$.

Let $f:[0, \omega] \times X \rightarrow \operatorname{Comp}(X)$ satisfy the following conditions.

$\left(\mathrm{H}_{1}\right) \quad f$ is $\mathscr{L} \otimes \mathscr{B}(X)$-measurable.

$\left(\mathrm{H}_{2}\right)$ There exists $k \in L^{1}([0, \omega], R)$ such that $h(f(t, x), f(t, y)) \leq k(t)|x-y|$, for all $x, y \in X$, a.e. in $[0, \omega]$.

$\left(\mathrm{H}_{3}\right)$ There is $\beta \in L^{\mathrm{l}}([0, \omega], R)$ such that $\operatorname{dist}(0, f(t, 0)) \leq \beta(t), t \in[0, \omega]$ a.e.

Consider the Cauchy problem

$$
x^{\prime} \in f(t, x), \quad x(0)=x_{0} .
$$

A map $x:[0, \omega] \rightarrow X$ is said to be a solution of $(5)$, if $x \in \mathrm{AC}([0, \omega], X)$ with $x(0)=x_{0}$ such that

$$
x^{\prime}(t) \in f(t, x(t)) \text { a.e. }
$$

The following theorem is vital to our discussion.

Continuous Selection theorem ([6, Theorem 3.1]). Suppose $f$ satisfies $\left(\mathrm{H}_{1}\right)$ $\left(\mathrm{H}_{3}\right)$. Then there exists a map $x:[0, \omega] \times X \rightarrow X$ such that

(I) for each $x_{0}, x\left(t, x_{0}\right)$ is a solution of (5) defined on $[0, \omega]$.

(II) The map $x_{0} \rightarrow x\left(\cdot, x_{0}\right)$ is continuous from $X$ into $\mathrm{AC}([0, \omega], X)$.

(III) $\left|x\left(t, x_{0}\right)-x_{0}\right| \leq \exp \left(\int_{0}^{t} k(s) d s\right)+\left|x_{0}\right|\left(\exp \left(\int_{0}^{t} k(s) d s\right)-1\right)$

$+\int_{0}^{t} \beta(\tau) \exp \left(\int_{\tau}^{t} k(s) d s\right) d \tau, t \in[0, \omega]$.

In the following, we also assume

$\left(\mathrm{H}_{4}\right)$ for each $x \in X, f(t+\omega, x)=f(t, x)$, for all $t \in R$.

Thus a solution $x(t)$ of $(4)$ defined on $R$ is said to be an $\omega$-periodic solution of (4) if $x(t+\omega)=x(t)$, for all $t \in R$.

Definition 1. Let $D \subset X$ be nonempty and bounded. Solutions of (4) are said to be uniformly compact for $D$, if there is an $S \in \operatorname{Comp}(X)$ such that for each $x_{0} \in D$, each solution $x(t)$ of (4) with initial data $x(0)=x_{0}$ satisfies

$$
x(t) \in S, \text { for } t>0 ;
$$

if given a bounded $D \subset X$, there is an $H>0$, such that for each $x_{0} \in X$ with $x_{0} \in D$, each solution $x(t)$ of $(4)$ with $x(0)=x_{0}$ satisfies

$$
|x(t)| \leq H \text { for } t \geq 0 \text {, }
$$

then solutions of (4) are said to be uniformly bounded for $D$.

Definition 2. Let $D_{1}, D_{2} \subset X$ be nonempty and bounded. Solutions of (4) are said to be uniformly $D_{1}-D_{2}$ bounded if there exists a $T>0$ such that for each $x_{0} \in D_{1}$, each solution $x(t)$ of $(4)$ with $x(0)=x_{0}$ satisfies

$$
x(t) \in D_{2} \text { for } t \geq T \text {. }
$$

Let $B>0$. If given any $M>0$, there is a $T=T(M)>0$ such that for each $x_{0} \in X$ with $\left|x_{0}\right| \leq M$, each solution $x(t)$ of (4) with $x(0)=x_{0}$ satisfies

$$
|x(t)| \leq B \text { for } t \geq T(M),
$$


then solutions of (4) are said to be uniformly ultimately bounded.

We are now in a position to state our main result.

Theorem 1. Let $D_{1}, D_{2} \subset X$ be nonempty, bounded, and convex such that $D_{2} \subset$ $D_{1}$, dist $\left(\partial D_{1}, D_{2}\right)>0$, and $D_{1}$ is open and $D_{2}$ is closed. If solutions of (4) are uniformly compact for $D_{1}$ and uniformly $D_{1}-D_{2}$ bounded, then (4) has an $\omega$ periodic solution lying in $D_{2}$.

To prove this theorem, we need the following asymptotic fixed point theorem.

HoRN's FIXED POINT THEOREM ([15]). Let $S_{0} \subset S_{1} \subset S_{2}$ be convex subsets of the Banach space $X$ such that $S_{0}$ and $S_{2}$ are closed and $S_{1}$ is open relative to $S_{2}$. Let $P: S_{2} \rightarrow X$ be continuous, compact, and such that for some integer $N>0$,

$$
P^{j}\left(S_{1}\right) \subset S_{2}, \quad 0 \leq j \leq N-1,
$$

and

$$
P^{j}\left(S_{1}\right) \subset S_{0}, \quad N \leq j \leq 2 N-1 .
$$

Then $P$ has a fixed point in $S_{0}$.

Proof of Theorem 1. By the uniform compactness of solutions for $D_{1}$, there is an $S \in \operatorname{Comp}(X)$ such that for each $x_{0} \in D_{1}$, each solution $x(t)$ of (4) with $x(0)=x_{0}$ satisfies

$$
x(t) \in S \text { for } t>0 \text {. }
$$

From the uniform $D_{1}-D_{2}$ boundedness of solutions it is known that there is a positive integer $N$ such that for each $x_{0} \in D_{1}$, each solution $x(t)$ of (4) with $x(0)=x_{0}$ satisfies

$$
x(t) \in D_{2} \quad \text { for } t \in[N \omega,(2 N-1) \omega] .
$$

According to the continuous selection theorem, there exists a map $x:[0,2 N \omega] \times$ $X \rightarrow X$ such that

(a) for each $x_{0} \in X, x\left(t, x_{0}\right)$ is a solution of (4) defined on $[0,2 N \omega]$;

(b) the map $x_{0} \rightarrow x\left(\cdot, x_{0}\right)$ is continuous from $X$ into $\mathrm{AC}([0,2 N \omega], X)$. Define the Poincare map $P: X \rightarrow X$ by

$$
P\left(x_{0}\right)=x\left(\omega, x_{0}\right),
$$

and set

$$
\begin{aligned}
& S_{2}=\overline{\mathrm{CO}}\left(\bigcup_{j=0}^{N}\left\{x\left(j \omega, x_{0}\right): x_{0} \in D_{1}\right\}\right) . \\
& S_{1}=D_{1}, \quad S_{0}=D_{2} .
\end{aligned}
$$

By (6) and the boundedness of $D_{1}, S_{2}$ is bounded, convex closed, and $P$ is continuous and compact. Obviously, $S_{0} \subset S_{1} \subset S_{2}$. Thus, using (6) and (7) we get

$$
\begin{array}{ll}
P^{j}\left(S_{1}\right) \subset S_{2}, & 0 \leq j \leq N-1, \\
P^{j}\left(S_{1}\right) \subset S_{0}, & N \leq j \leq 2 N-1 .
\end{array}
$$

By Horn's fixed point theorem, $P$ has a fixed point $x^{*} \in S_{0}$, which implies that

$$
x\left(\omega, x^{*}\right)=x\left(0, x^{*}\right) .
$$


Define

$$
\tilde{x}(t)= \begin{cases}x\left(t, x^{*}\right), & t \in[0, \omega], \\ x\left(t-j \omega, x^{*}\right), & t \in[j \omega,(j+1) \omega], j= \pm 1, \pm 2, \ldots\end{cases}
$$

Then $\tilde{x}(t)$ is an $\omega$-periodic solution of (4), which completes the proof.

3. Equilibrium states. Now we answer the existence problem of equilibria for (3) by the following theorem.

THEOREM 2. Let the following conditions be fulfilled.

(I) $F: \mathbf{R}^{n} \rightarrow \operatorname{Comp}\left(\mathbf{R}^{n}\right)$ is a Lipschitz map, that is, for each $x \in \mathbf{R}^{n}$ there exist $k, r>0$ such that $h(F(y), F(z)) \leq k(x)|y-z|$ for $y, z \in S(x, r)$, where $S(x, r)=\left\{y \in \mathbf{R}^{n}: \operatorname{dist}(y, x)<r\right\}$.

(II) Given any $D \in \operatorname{Comp}\left(\mathbf{R}_{+}^{n}\right)$, solutions of (3) are uniformly bounded for $D$.

(III) There are $M>m>0$ such that given any $D \in \operatorname{Comp}\left(\mathbf{R}_{+}^{n}\right)$, there is a $T>0$ such that for each $x_{0} \in D$, each solution $x(t)$ of (3) satisfies

$$
m \leq x_{i}(t) \leq M, \quad t \geq T \text {. }
$$

Then (3) has an equilibrium in $\operatorname{int}\left(\mathbf{R}_{+}^{n}\right)$, i.e., there is an $x^{*} \in \mathbf{R}_{+}^{n}$ such that

$$
0 \in F\left(x^{*}\right) \text {. }
$$

Proof. Set

$$
\begin{aligned}
& D_{1}=\left\{y \in \mathbf{R}^{n}: 0 \leq y_{i} \leq 2 m, i=1, \ldots, n\right\}, \\
& D_{2}=\left\{y \in \mathbf{R}^{n}: \frac{m}{2} \leq y_{i} \leq \frac{3 M}{2}, i=1, \ldots, n\right\} .
\end{aligned}
$$

By Theorem 1, given any $\omega>0,(3)$ has an $\omega$-periodic solution $x\left(t, x_{0}(\omega)\right)$ satisfying

$$
\frac{m}{2} \leq x_{i}\left(t, x_{0}(\omega)\right) \leq \frac{3 M}{2}, \quad t \in \mathbf{R}, i=1, \ldots, n .
$$

Applying [17, Lemma 2], we know that there is $x^{*} \in \mathbf{R}^{n}$ with $\frac{m}{2} \leq x_{i}^{*} \leq \frac{3 M}{2}, i=$ $1, \ldots, n$ such that

$$
x\left(t, x^{*}\right) \equiv x^{*}, \quad t \in \mathbf{R} .
$$

By the continuous selection theorem (I), $x\left(t, x^{*}\right)$ is a solution of (3). Hence

$$
0 \in F\left(x^{*}\right) \text {, }
$$

which proves the theorem.

Acknowledgment. The authors thank the referee for his very helpful comments.

\section{REFERENCES}

[1] T. F. Bridgland, Jr., Contributions to the theory of generalized differential equations I, Math. Systems Theory 3, 17-50 (1969)

[2] F. E. Browder, On a generalization of the Schauder fixed point theorem, Duke Math. J. 26, 291-303 (1959)

[3] T. A. Burton and V. Hutson, Repellers in systems with infinite delay, J. Math. Anal. Appl. 137, 240-263 (1989) 
[4] T. A. Burton and S. Zhang, Unified boundedness, periodicity, and stability in ordinary and functional differential equations, Ann. Mat. Pura Appl. CXLV, 129-258 (1986)

[5] G. Butler, H. Freedman, and P. Waltman, Uniformly persistent systems, Proc. Amer. Math. Soc. 96, 425-430 (1986)

[6] R. M. Colombo, A. Fryszkowski, T. Rzežuchowski, and V. Staicu, Continuous selections of solutions sets of Lipschitzean differential inclusions, Funkcial Ekvac. 34, 321-330 (1991)

[7] H. Freedman and J. So, Persistence in discrete semi-dynamical systems, SIAM J. Math. Anal. 20, 930-938 (1989)

[8] R. E. Gaines, R. B. Guenther, and J. W. Lee, Some existence results for differential inclusions, C. R. Acad. Sci. Paris 307, 391-396 (1988)

[9] R. E. Graines and J. K. Peterson, Periodic solutions to differential inclusions, Nonlinear Analysis 5, 1109-1131 (1981)

[10] G. Haddad and M. Lasry, Periodic solutions of functional differential inclusions and fixed points of $\sigma$-selectionable correspondences, J. Math. Anal. Appl. 96, 295-312 (1983)

[11] J. K. Hale and O. Lopes, Fixed point theorem and dissipative processes, J. Differential Equations 13, 391-402 (1973)

[12] J. Hofbauer, An index theorem for dissipative semiflows, Rocky Mount. J. Math. 20, 1017-1031 (1990)

[13] J. Hofbauer, V. Hutson, and W. Jansen, Coexistence for systems governed by difference equations of Lotka-Volterra type, J. Math. Biol. 25, 553-570 (1987)

[14] J. Hofbauer and K. Sigmund, Dynamical Systems and the Theory of Evolution, Cambridge University Press, 1988

[15] W. A. Horn, Some fixed point theorem for compact maps and flows in Banach spaces, Trans. Amer. Math. Soc. 149, 391-404 (1970)

[16] V. Hutson, $A$ theorem on average Liapunov functions, Monatsh. Math. 98, 267-275 (1984)

[17] V. Hutson, The existence of an equilibrium for permanent systems, Rocky Mountain J. Math. 20, 1033-1040 (1990)

[18] V. Hutson and W. Moran, Persistence of species obeying difference equations, Math. Biosci. 15, 203-213 (1982)

[19] V. Hutson and W. Moran, Repellers in reaction-diffusion systems, Rocky Mountain J. Math. 17, 301-314 (1987)

[20] W. G. Kelley, Periodic solutions of generalized differential equations, SIAM J. Appl. Math. 30, 70-74 (1976)

[21] Li Yong, Wang Huaizhong, and Lü Xianrui, Equilibrium of permanent multivalued systems, Quart. Appl. Math. 51, 791-795 (1993)

[22] L. E. Miller, Generalized boundary-value problems, J. Math. Anal. Appl. 74, 233-246 (1980)

[23] Jack W. Macki, P. Nistri, and P. Zecca, The existence of periodic solutions to nonautonomous differential inclusions, Proc. Amer. Math. Soc. 104, 840-844 (1988)

[24] R. Nussbaum, Some asymptotic fixed point theorems, Trans. Amer. Math. Soc. 177, 349-375 (1972)

[25] S. Plaskacz, Periodic solutions of nonlinear functional differential inclusions on compact subsets of $\mathbf{R}^{n}$, J. Math. Anal. Appl. 148, 202-212 (1990)

[26] V. Staicu, Continuous selections of solutions sets to evolution equations, Proc. Amer. Math. Soc. 113, 403-413 (1991)

[27] T. Yoshizawa, Stability Theory by Liapunov's Second Method, Math. Soc. Japan, Tokyo, 1966

[28] S. Zaremba, Sur les équations au paratingent, Bull. Sci. Math. 60, 139-160 (1963) 